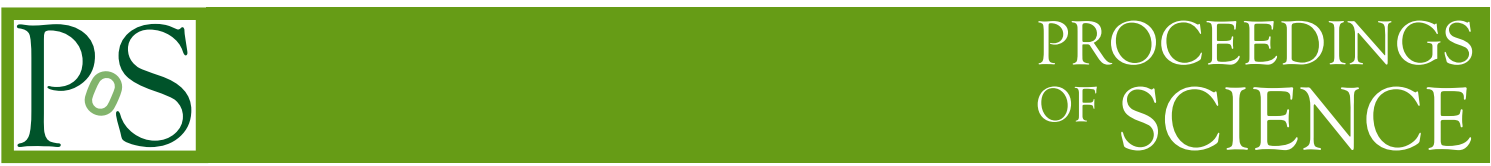

\title{
GRB Studies with AGILE
}

\section{Francesco Longo $^{* a, b}$, A. Giuliani ${ }^{c}$, F. Verrecchia ${ }^{d, e}$, C. Pittori ${ }^{d, e}$, M. Marisaldi $^{f}$, F. Fuschino ${ }^{f}$, E. Del Monte ${ }^{g}$, A. Rappoldi ${ }^{h}$ on behalf of the AGILE team}

${ }^{a}$ Dipartimento di Fisica, Università di Trieste, via A. Valerio 2, I-34127 Trieste, Italy

${ }^{b}$ INFN Trieste, via A. Valerio 2, I-34127 Trieste, Italy

${ }^{c}$ INAF-IASF Milano, via E. Bassini 15, I-20133 Milano, Italy

${ }^{d}$ ASI Science Data Center, Via del Politecnico snc, I-00133 Roma, Italy

${ }^{e}$ INAF-OAR, Roma, via di Frascati 33, I-00040 Monte Porzio Catone, Italy

${ }^{f}$ INAF-IASF Bologna, via Gobetti 101, I-40129 Bologna, Italy

g INAF-IAPS Roma, via del Fosso del Cavaliere 100, I-00133 Roma, Italy

${ }^{h}$ INFN Pavia, via Bassi 6, I-27100 Pavia, Italy

E-mail: francesco.longodts.infn.it

The AGILE satellite, in orbit since 2007, is detecting about one Gamma Ray Burst (GRB) per week with the non-imaging Mini-Calorimeter (MCAL, 0.35 - $100 \mathrm{MeV}$ ), and localized about one GRB per month with the hard X-ray imager SuperAGILE (18 - $60 \mathrm{keV})$ when operated in pointing observing mode (up to October 2009), with a rate reduced by a factor 2-3 in spinning observing mode (up to December 2011). Up to now the AGILE Gamma Ray Imaging Detector (GRID) firmly detected eight GRBs in the energy band between $20 \mathrm{MeV}$ and few $\mathrm{GeV}$. In this paper we review the status of the GRB observations with AGILE-GRID instrument, and discuss the upper limits in the gamma-ray band for the non-detected events.

Science with the New Generation of High Energy Gamma-ray experiments, 10th Workshop 04-06 June 2014

Lisbon - Portugal

\footnotetext{
* Speaker.
} 


\section{The AGILE satellite}

Launched on April 2007, AGILE [回], [2] is a scientific satellite included in the "Small mission" program of the Italian Space Agency (ASI) operating at a low inclination $\left(2.5^{\circ}\right)$ Low-Earth Orbit at $540 \mathrm{~km}$ altitude and is devoted to the observation of the sky in the hard X-ray and gamma-ray energy bands .

The AGILE payload is composed of two co-aligned imagers: the hard X-ray monitor SuperAGILE [3], sensitive in the 18-60 keV energy band with a field of view of $\sim 1 \mathrm{sr}$ and an angular resolution of 6 arcmin, and the Gamma Ray Imaging Detector (GRID, [П]), a pair-tracking telescope based on a tungsten-silicon tracker [5], sensitive in the energy band from $30 \mathrm{MeV}$ to few $\mathrm{GeV}$ with a field of view of $\sim 2.5 \mathrm{sr}$ and a point spread function ranging between $3.5^{\circ}$ (at $100 \mathrm{MeV}$ ) and $0.7^{\circ}$ (at $1 \mathrm{GeV}$ ). The Minicalorimeter (MCAL, [6]) of the gamma-ray imager, based on CsI(Tl) scintillating bars, can independently detect transient events at $\mathrm{MeV}$ energies, using a dedicated trigger logic acting on several time scales spanning four orders of magnitude between $290 \mu$ s and 8 seconds 何, in an almost all-Sky field of view with maximum sensitivity at an angle of roughly $90^{\circ}$ with respect to the satellite boresight.

\section{Observation of GRB with AGILE}

Both SuperAGILE and MCAL are equipped with on-board triggering algorithms (see [8] and [7] respectively) developed to detect short gamma-ray transient events such as Gamma Ray Bursts (GRBs) and Terrestrial Gamma-ray Flashes. Dedicated telemetry packets are introduced in the data stream to downlink the trigger information. Since the beginning of the AGILE operations, SuperAGILE and MCAL are active members of the InterPlanetary Network (IPN). On November 2009 AGILE suffered a malfunction to the reaction wheel and, after that time, the satellite is working in a spinning operative mode, with an angular velocity of $\sim 0.8^{\circ}$ per second around the axis pointing toward the Sun and scanning $\sim 70 \%$ of the Sky in every orbit. In pointing operative mode the localization rate of SuperAGILE was $\sim 1$ GRB per month and it was reduced by a factor of $2-3$ in spinning mode. While all AGILE instruments functions are still nominal, due to ASI's Malindi ground station problems in 2012 the number of AGILE daily passes acquired from ground has been decreased from 16-17/day to 6-7/day on average, and SuperAGILE was put in the so-called "Idle" mode since September 2012 to optimize the AGILE-GRID telemetry budget. The last GRB detected by SuperAGILE is GRB 111211A [9], located at a redshift of 0.478 [10] and associated with a Supernova [11]. The MCAL detection rate is $\sim 1$ GRB per week and is pratically not affected by the spinning operative mode. All the 85 hard GRB detected by MCAL in the pointing mode until October 2009 were analysed in detail. The resulting catalog was recently published cointaining timing data for 84 and spectral data for 21 bursts[12], and it is also available from the AGILE Data Center web pages in the form of an interactive web table ${ }^{1}$ providing some additional information.

Until September 2014 the GRID firmly detected eight GRBs in the high-energy gammaray band: GRB 080514B [13], [14], GRB 090401B [15], GRB 090510 [16], [17], [18], GRB 100724B [19], GRB130327B[20], GRB 130427A[21], GRB 131108A[22] and GRB 140330A[23].

\footnotetext{
${ }^{1}$ http: //www.asdc.asi.it/mcalgrbcat/
} 
Two additional GRBs (GRB 080721 and GRB 081001) showed lower significant detections in gamma-rays.

The GRB detected by AGILE-GRID show all the relevant features in the high-energy gammaray band as currently detected also by Fermi-LAT (e.g. [24]), such as the delayed emission with respect to lower energies as in the short GRB 090510 [16] where the gamma-ray emission started after $0.2 \mathrm{~s}$ and when the bulk of low-energy emission detected by AGILE-MCAL was ended; the presence of spectral extra-components as in the case of 131108A [22] where the high energy extracomponent is significantly detected during the prompt phase; the extended emission, again in the case of the remarkable short GRB 090510 [16] with emission extended up to $\sim 10 \mathrm{~s}$ with a temporal decay with index $\alpha \sim-1.3$ but also in GRB 080514B and GRB 090401B, even with different characteristics, with the latter having more photons in the prompt phase differently from the former, and an example of the so called "super-long" GRB events in the case of GRB 130427A [21, 25] that was detected by AGILE-GRID over a period of 12 hours. Another interesting case is GRB 100724B where the low energy and the high-energy emission seem to be correlated in time, contrary to the majority of GRBs in the high-energy band[19]. These few cases demonstrate the great variety of gamma-ray emission properties of GRBs and the need of a better understanding of the physical mechanisms generating such features.

The large field of view of the GRID allows to observe simultaneously about one fifth of the sky. Motivated by the fact that significant gamma-ray emission is observed from only a small fraction of GRBs, corresponding to few events per year, taking into account the AGILE and FermiLAT detections, we estimated the upper limits on the flux of GRBs within the GRID field of view between July 2007 and October 2009, localised by SuperAGILE, Swift/BAT, INTEGRAL/IBIS, Fermi/GBM and IPN. Our sample is composed of 68 bursts, of which 40 have spectral information, publicly distributed through the GCN Circulars by Konus-Wind, Suzaku/WAM and Fermi/GBM. We estimated the upper limits using a Bayesian approach and following the method proposed by [26] and [27]. When available, the spectral model from the publicly available information is used to calculate the flux upper limit and to extrapolate the available flux to the energy band between 30 $\mathrm{MeV}$ and $3 \mathrm{GeV}$. In the other cases, we adopted for these calculations the average values measured by BATSE for the photon index of an exponential cutoff and for the high energy photon index of a Band function. We found that the calculated upper limits are constraining the extrapolation of the Band spectrum in $\sim 10 \%$ of the GRB sample[28]. The consequences of such constraints on the emission models allow to estimate upper limits on the bulk Lorentz factor $\Gamma_{0}$ in the external shocks scenario[29], and to provide estimates of some of the microphysical parameters of the shocks in the internal shock scenario. A remarkable case of this behaviour is the long GRB 090618, localised by Swift [30] and also by SuperAGILE (in a consistent position) and detected by the MCAL [31]. The GRB has a steep spectrum as measured by MCAL in the energy band between $350 \mathrm{keV}$ and 100 $\mathrm{MeV}$, with a photon index of -3.16 [31] and therefore is not detected in gamma-rays by the GRID.

Moreover, in our analysis we investigated the GRID data of the sample of GRBs within our field of view and we did not find any significant gamma-ray afterglow emission until $3600 \mathrm{~s}$ after trigger [28]. 


\section{Conclusions}

The ongoing observation of GRBs with AGILE and Fermi is showing that only a small subsample of events emits in gamma rays above $30 \mathrm{MeV}$ during the prompt phase. In fact, the overall detection rate taking into account both satellites is around 10 bursts per year, consistent with current estimates [32]. Future analysis techniques or higher sensitive detectors in the energy band (10 $\mathrm{MeV}-100 \mathrm{MeV}$ ) might clarify the presence of spectral cut-offs in the high-energy component of the prompt emission.

\section{References}

[1] M. Tavani et al., Nucl. Inst. and Meth. A, 588, 52 (2008)

[2] M. Tavani et al., $A \& A, \mathbf{5 0 2}, 995$ (2009)

[3] M. Feroci et al., Nucl. Inst. and Meth. A, 581, 728 (2007)

[4] M. Prest et al., Nucl. Instr. and Meth. A, 501, 280 (2003)

[5] A. Bulgarelli et al., Nucl. Instr. and Meth. A, 614, 213 (2010)

[6] C. Labanti, et al., Nucl. Instr. and Meth. A, 598, 470 (2009)

[7] F. Fuschino, et al., Nucl. Instr. and Meth. A, 588, 17 (2008)

[8] E. Del Monte et al., in Proceedings of SciNeGHE 2007, edited by A. Lionetto and A. Morselli, Frascati Physics Series, 45, 201, (2007)

[9] F. Lazzarotto et al., GCN Circular, 12666 (2011)

[10] S. Vergani et al., GCN Circular, 12677 (2011)

[11] A. de Ugarte Postigo et al., GCN Circular, 12802 (2011)

[12] M. Galli et al., $A \& A, \mathbf{5 3 3}, \mathrm{A} 13$ (2013)

[13] A. Giuliani et al., A\&A, 491, L25 (2008)

[14] A. Rossi et al., A\&A, 491, L29 (2008)

[15] E. Moretti et al., GCN Circular, 9069 (2009)

[16] A. Giuliani et al., ApJL, 708, L84 (2010)

[17] M. Ackerman et al., ApJ, 716, 1178 (2010)

[18] M. De Pasquale et al., ApJL, 709, L146 (2010)

[19] E. Del Monte et al., A\&A, 535, A120, (2011)

[20] F. Longo et al.,GCN Circular, 14344 (2013)

[21] F. Verrecchia et al., GCN Circular, 14515 (2013)

[22] A. Giuliani et al., submitted to $A \& A$, (2014) [http://arxiv.org/abs/1407.0238]

[23] C. Pittori et al.,GCN Circular, 16058 (2013)

[24] M. Ackermann et al.,ApJS, 209, 11 (2013)

[25] M. Ackermann et al,, Science, 343, 42 (2014) 
[26] O. Helene, Nucl. Instr. and Meth. A, 212, 319 (1983)

[27] O. Helene, Nucl. Instr. and Meth. A, 228, 120 (1984)

[28] F. Longo, et al., $A \& A$ 547, A95 (2012)

[29] G. Ghisellini et al., MNRAS 403, 926 (2010)

[30] P. Schady et al., GCN Circular, 9512 (2009)

[31] F. Longo et al., GCN Circular, 9524 (2009)

[32] D. Band et al., ApJ, 701, 1673 (2009) 